\title{
An Optimal Control Modification to Model-Reference Adaptive Control for Fast Adaptation
}

\author{
Nhan T. Nguyen* \\ Kalmanje Krishnakumar ${ }^{\dagger}$ \\ NASA Ames Research Center, Moffett Field, CA 94035 \\ Jovan Boskovic \\ Scientific Systems Company, Inc., Boston, MA 01801
}

\begin{abstract}
This paper presents a method that can achieve fast adaptation for a class of model-reference adaptive control. It is well-known that standard model-reference adaptive control exhibits high-gain control behaviors when a large adaptive gain is used to achieve fast adaptation in order to reduce tracking error rapidly. Highgain control creates high-frequency oscillations that can excite unmodeled dynamics and can lead to instability. The fast adaptation approach is based on the minimization of the squares of the tracking error, which is formulated as an optimal control problem. The necessary condition of optimality is used to derive an adaptive law using the gradient method. This adaptive law is shown to result in uniform boundedness of the tracking error by means of the Lyapunov's direct method. Furthermore, this adaptive law allows a large adaptive gain to be used without causing undesired high-gain control effects. The method is shown to be more robust than standard model-reference adaptive control. Simulations demonstrate the effectiveness of the proposed method.
\end{abstract}

\section{Introduction}

In recent years, adaptive control has been receiving a significant amount of attention. The Aviation Safety Program under the NASA Aeronautics Research Mission Directorate (ARMD) has established the Integrated Resilient Aircraft Control (IRAC) research project to advance the state of the arts in adaptive control to enable flight control resiliency in the presence of adverse conditions. ${ }^{1}$ There has been a steady increase in the number of adaptive control applications in a wide range of settings such as aerospace, robotics, process control, etc. Research in adaptive control continues to receive attention from government agencies, industries, and academia. In aerospace applications, adaptive control have been developed for many flight vehicles. For example, NASA has recently conducted a flight test in 2006 of a neural net intelligent flight control system on board of a modified F-15 test aircraft. ${ }^{2}$ The U.S. Air Force-Boeing team has successfully developed and completed numerous flight tests of direct adaptive control on Joint Direct Attack Munitions (JDAM). ${ }^{3}$ The ability to accommodate system uncertainties and to improve fault tolerance of a flight control system is a major selling point of adaptive control. Nonetheless, adaptive control still faces significant challenges in providing robustness in the presence of unmodeled dynamics and parametric uncertainties. The crash of the X-15 aircraft in $1967^{4}$ serves as a reminder that adaptive control is still viewed with some misgivings despite enormous advances in this technology ever since. The ability for an adaptive control algorithm to modify a pre-existing control design is considered a strength and at the same time a weakness.

Over the past several years, various model-reference adaptive control (MRAC) methods have been investigated. ${ }^{5-11,13,14}$ The majority of MRAC methods may be classified as direct, indirect, or a combination thereof. Indirect adaptive control methods are based on identification of unknown plant parameters and certainty-equivalence control schemes derived from the parameter estimates which are assumed to be their true values. ${ }^{17}$ Parameter identification techniques such as recursive least-squares and neural networks have been used in indirect adaptive control methods. ${ }^{7}$ In contrast, direct adaptive control methods directly adjust control parameters to account for system uncertainties without identifying unknown plant parameters explicitly. MRAC methods based on neural networks has been a topic of great research interests. $^{8-10}$ In particular, Rysdyk and Calise described a neural net direct adaptive control method for improving

\footnotetext{
${ }^{*}$ Research Scientist, Intelligent Systems Division, Mail Stop 269-1, AIAA Senior Member

${ }^{\dagger}$ Research Scientist, Intelligent Systems Division, Mail Stop 269-1, AIAA Associate Fellow

${ }^{\ddagger}$ Principal Research Engineer \& Group Leader, Intelligent \& Autonomous Control Systems, AIAA Senior Member
} 
tracking performance. ${ }^{8}$ This method is the basis for the intelligent flight control system that has been developed for the F-15 test aircraft by NASA. Johnson et al. introduced a pseudo-control hedging approach for dealing with control input characteristics such as actuator saturation, rate limit, and linear input dynamics. ${ }^{10}$ Idan et al. studied a hierarchical neural net adaptive control using secondary actuators such as engine propulsion to accommodate for failures of primary actuators. ${ }^{11}$ Hovakimyan et al. developed an output feedback adaptive control to address issues with parametric uncertainties and unmodeled dynamics. ${ }^{14}$ Cao et al. developed an $\mathscr{L}_{1}$ adaptive control method to address high-gain control. $^{12}$

While adaptive control has been used with success in a number of applications, the possibility of high-gain control due to fast adaptation can be an issue. In certain applications, fast adaptation is needed in order to improve tracking performance when a system is subject to a large source of uncertainties such as structural damage to an aircraft that could cause large changes in aerodynamic derivatives. In these situations, a large adaptive gain or learning rate must be used in the adaptive control in order to reduce the tracking error rapidly. However, there typically exists a balance between stability and adaptation. It is well-known that fast adaptation can result in high-frequency oscillations which can excite unmodeled dynamics that could adversely affect the stability of an MRAC law. Recognizing this, some recent adaptive control methods have begun to address high-gain control, such as the $\mathscr{L}_{1}$ adaptive control ${ }^{12}$ and a hybrid direct-indirect adaptive control. ${ }^{15}$ In the former approach, the use of a low-pass filter effectively prevents any high frequency oscillation that may occur due to fast adaptation. In so doing, the reference model is no longer preserved and instead must be reconstructed using a predictor model. In the latter approach, an indirect adaptive law based on a recursive least-squares parameter estimation adjusts the parameters of a nominal controller to reduce the modeling error, and the remaining tracking error signal could then be handled by a direct adaptive law with a less aggressive learning rate.

This paper introduces a new approach to fast adaptation in the MRAC framework. The method is formulated as an optimal control problem to minimize the tracking error $\mathscr{L}_{2}$-norm. The optimality condition results in a modification to the MRAC law by introducing a damping term proportional to persistent excitation. The optimal control modification is then analyzed to determine convergence and stability characteristics. The analysis shows that this modification can achieve fast adaptation without high-frequency oscillations as in the case with the standard MRAC. Furthermore, the modification is shown to provide improved stability robustness while preserving the tracking performance.

\section{Optimal Control Modification for Fast Adaptation}

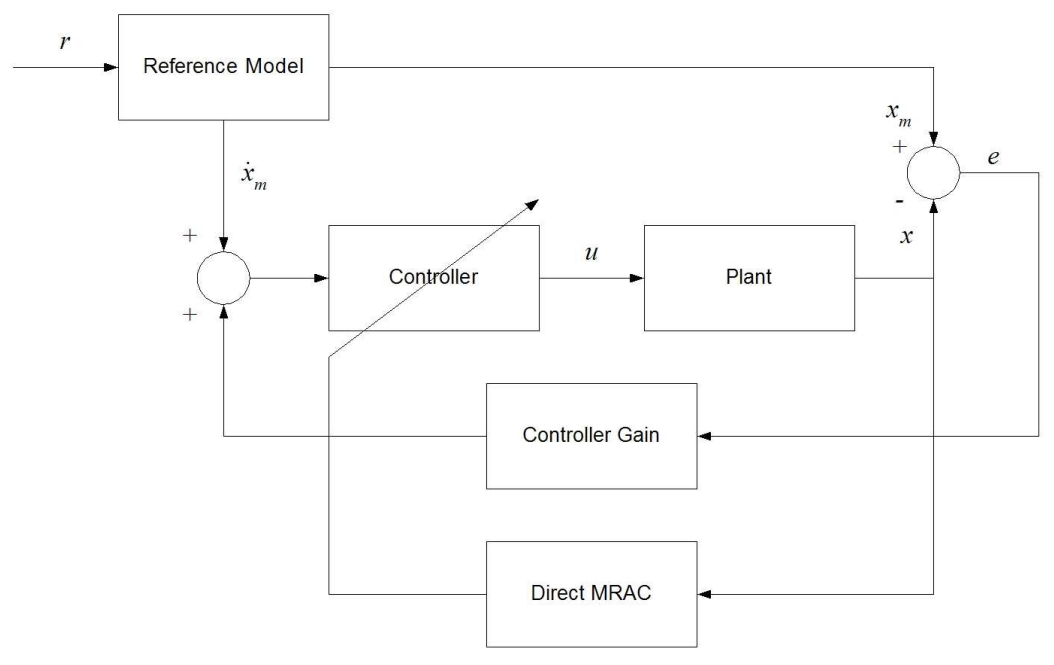

Fig. 1 - Direct MRAC

A direct MRAC problem as illustrated in Fig. 1 is posed as follows:

Given a nonlinear plant model as

$$
\dot{x}=A x+B[u+f(x)]
$$

where $x(t):[0, \infty) \rightarrow \mathbb{R}^{n}$ is a state vector, $u(t):[0, \infty) \rightarrow \mathbb{R}^{p}$ is a control vector, $A \in \mathbb{R}^{n \times n}$ and $B \in \mathbb{R}^{n \times p}$ are known plant matrices such that the pair $(A, B)$ is controllable, and $f(x): \mathbb{R}^{n} \rightarrow \mathbb{R}^{p}$ is a matched uncertainty that acts as a disturbance. 
Assumption 1: $x(t) \in C^{i}(t)$ is smooth in $t \in[0, \infty)$.

Assumption 2: $f(x) \in C^{1}(x)$ is semi-globally Lipschitz. Then there exists a constant $L>0$ such that

$$
\left\|f(x)-f\left(x_{0}\right)\right\| \leq L\left\|x-x_{0}\right\|
$$

for all $\|x\|_{\infty}<\varepsilon_{x}$ in $t \in[0, \infty)$.

It then follows that the partial derivatives of $f(x)$ are uniformly bounded and at least piecewise continuous such that

$$
\left\|\frac{\partial f(x)}{\partial x}\right\|_{\infty} \leq L
$$

for all $\|x\|_{\infty}<\varepsilon_{x}$ in $t \in[0, \infty)$.

Proposition 1: If $u(t)$ is a stable and bounded controller, then the total derivative of $f(x)$ is also bounded.

Proof: $u(t)$ is bounded if there exists a constant $\varepsilon_{u}>0$ such that $\|u\|_{\infty}<\varepsilon_{u} \forall t \in[0, \infty) . u(t)$ is a stable controller which implies that $x(t)$ is bounded and so $\|x\|_{\infty}<\varepsilon_{x} \forall t \in[0, \infty)$. Since $x(t)$ is at least $C^{1}$ smooth by Assumption 1 , $x(t)$ is bounded, $f(x)$ is semi-globally Lipschitz, and if $u(t)$ is bounded, then $\dot{x}(t)$ is also bounded. Thus, there exists a constant vector $\sigma_{x_{i}}>0 \in \mathbb{R}, i=1, \ldots n$, such that $\sup _{t}\left|\dot{x}_{i}\right| \leq \sigma_{x_{i}} \forall t \in[0, \infty)$. It then follows that

$$
\sup _{t}\left|\frac{d f(x)}{d t}\right| \leq\left\|\frac{\partial f(x)}{\partial x}\right\|_{\infty} \mathscr{I} \sum_{i=1}^{n} \sup _{t}\left|\dot{x}_{i}\right| \leq L \mathscr{I} \sum_{i=1}^{n} \sigma_{x_{i}}=\sigma_{f}
$$

for some $\sigma_{f}>0 \in \mathbb{R}^{p}$, where $\mathscr{I} \in \mathbb{R}^{p}$ is a vector whose elements are all equal to one. Therefore, $\dot{f}(x) \in \mathscr{L}_{\infty}$.

The objective of the problem is to design a full-state feedback controller that enables the nonlinear plant model to follow a reference model described by

$$
\dot{x}_{m}=A_{m} x_{m}+B_{m} r
$$

where $A_{m} \in \mathbb{R}^{n \times n}$ is Hurwitz and a known matrix, $B_{m} \in \mathbb{R}^{n \times p}$ is also a known matrix, and $r(t):[0, \infty) \rightarrow \mathbb{R}^{p} \in \mathscr{L}_{\infty}$ is a bounded command vector with its time derivative $\dot{r} \in \mathscr{L}_{\infty}$ also bounded.

Defining the tracking error as $e=x_{m}-x$, the goal is then to determine a controller that results in $\lim _{t \rightarrow \infty}\|e\| \leq \varepsilon_{e}$. Toward that end, let the controller be comprised of a state feedback, a command feedforward, and an adaptive signal as follows:

$$
u=K_{e} e+K_{m} x_{m}+K_{r} r-u_{a d}
$$

where $K_{e} \in \mathbb{R}^{p \times n}, K_{m} \in \mathbb{R}^{p \times n}$, and $K_{r} \in \mathbb{R}^{p \times p}$ are known nominal gain matrix, and $u_{a d} \in \mathbb{R}^{p}$ is a direct adaptive signal.

Then, the tracking error dynamics become

$$
\dot{e}=\dot{x}_{m}-\dot{x}=\left(A-B K_{e}\right) e+\left(A_{m}-A-B K_{m}\right) x_{m}+\left(B_{m}-B K_{r}\right) r+B\left[u_{a d}-f(x)\right]
$$

For bounded tracking error, we choose $A_{c}=A-B K_{e}=A_{m}$, and the gain matrices $K_{m}$ and $K_{r}$ to satisfy the model matching conditions so that the nominal plant tracks the reference model

$$
\begin{gathered}
A-B K_{e}=A_{m} \\
A+B K_{m}=A_{m} \\
B K_{r}=B_{m}
\end{gathered}
$$

The adaptive signal $u_{a d}$ can be parameterized by a linear-in-parameters matched uncertainty

$$
u_{a d}=\Theta^{\top} \Phi(x)
$$

where $\Theta \in \mathbb{R}^{m \times p}$ is a weight matrix and $\Phi(x): \mathbb{R}^{n} \rightarrow \mathbb{R}^{m}$ is a known regressor vector.

Let $\Theta^{*}$ be a constant ideal weight matrix and $\tilde{\Theta}=\Theta-\Theta^{*}$ is a weight variation, then $\varepsilon$ is the approximation error defined as

$$
\varepsilon(x)=\Theta^{* \top} \Phi-f(x)
$$

Assumption 3: The approximation error $\varepsilon(x)$ of the matched uncertainty $f(x)$ by $\Theta^{\top} \Phi(x)$ is bounded and its time derivative is also bounded; i.e., there exists a constant vector $\sigma_{\varepsilon}>0 \in \mathbb{R}^{p}$ such that

$$
\sup _{t}|\dot{\varepsilon}(x)|=\sup _{t}\left|\frac{d\left(\Theta^{* \top} \Phi\right)}{d t}-\frac{d f(x)}{d t}\right|<\sigma_{e}
$$


Assumption 3 essentially implies that $\Phi$ is Lipschitz and its partial derivative is bounded, i.e., there exist constants $C>0$ and $\eta>0$ such that

$$
\begin{gathered}
\left\|\Phi(x)-\Phi\left(x_{0}\right)\right\| \leq C\left\|x-x_{0}\right\| \\
\left\|\frac{\partial \Phi(x)}{\partial x}\right\| \leq \eta
\end{gathered}
$$

for all $\|x\|_{\infty}<\varepsilon_{x}$ in $t \in[0, \infty)$.

The tracking error dynamics can now be expressed as

$$
\dot{e}=A_{c} e+B\left(\tilde{\Theta}^{\top} \Phi+\varepsilon\right)
$$

Defining $\delta_{e}=\sup _{t}|\varepsilon|$ as an upper bound of $\varepsilon$, then

$$
\dot{e} \leq A_{c} e+B\left(\tilde{\Theta}^{\top} \Phi+\delta_{e}\right)
$$

An optimal control modification to MRAC for fast adaptation is proposed as follows:

Proposition 2: The following adaptive law provides a weight update law that minimizes the $\mathscr{L}_{2}$-norm of the tracking error:

$$
\dot{\Theta}=-\Gamma \Phi\left(e^{\top} P-v \Phi^{\top} \Theta B^{\top} P A_{c}^{-1}\right) B
$$

where $\Gamma>0 \in \mathbb{R}^{m \times m}$ is a symmetric positive-definite learning rate or adaptive gain matrix, $v>0 \in \mathbb{R}$ is a positive weighting constant, and $P>0 \in \mathbb{R}^{n \times n}$ is a symmetric positive-definite matrix $P>0$ that solves the Lyapunov equation

$$
P A_{c}+A_{c}^{\top} P=-Q
$$

where $Q>0 \in \mathbb{R}^{n \times n}$ is a symmetric positive-definite matrix.

Proof: The adaptive law seeks a solution that minimizes the $\mathscr{L}_{2}$-norm of the tracking error with a cost function

$$
J=\frac{1}{2} \int_{0}^{t_{f}}(e-\Delta)^{\top} Q(e-\Delta) d t
$$

subject to Eq. (17) where $\Delta$.represents the tracking error at $t=t_{f}$.

$J$ is convex and represents the distance measured from the normal surface of a ball $B_{r}$ with a radius $\Delta$.

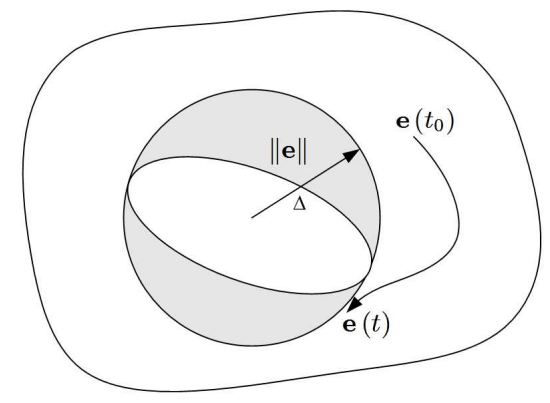

Fig. 2 - Tracking Error Bound

This is an optimal control problem whose solution can be formulated by the Pontryagin's Maximum Principle. Defining a Hamiltonian

$$
H\left(e, \tilde{\Theta}^{\top} \Phi\right)=\frac{1}{2}(e-\Delta)^{\top} Q(e-\Delta)+p^{\top}\left(A_{c} e+B \tilde{\Theta}^{\top} \Phi+B \delta_{e}\right)
$$

where $p \in \mathbb{R}^{n}$ is an adjoint variable, then the adjoint equation is given by the negative gradient of the Hamiltonian with respect to the tracking error

$$
\dot{p}=-\nabla H_{e}^{\top}=-Q(e-\Delta)-A_{c}^{\top} p
$$


Treating $\tilde{\Theta}^{\top}$ as a control variable, then the optimality condition is obtained by the gradient of the Hamiltonian with respect to $\widetilde{\Theta}^{\top} \Phi$

$$
\nabla H_{\tilde{\Theta}^{\top} \Phi}=p^{\top} B
$$

The adaptive law can then be formulated by the gradient method as

$$
\dot{\tilde{\Theta}}=-\Gamma \Phi \nabla H_{\tilde{\Theta}^{\top} \Theta}=-\Gamma \Phi p^{\top} B
$$

where $\Gamma>0$ is an adaptive gain or learning rate matrix.

If $e(0)$ is known, then the transversality condition requires

$$
p\left(t_{f}\right)=0
$$

This results in a two-point boundary value problem whereby the adjoint $p$ solves Eqs. (22) and (17) simultaneously. The optimal control problem can be solved using a "sweeping" method by letting $p=P e+S \Theta^{\top} \Phi$. Then

$$
\dot{P} e+P\left(A_{c} e+B \Theta^{\top} \Phi-B \Theta^{* \top} \Phi+B \delta_{e}\right)+\dot{S} \Theta^{\top} \Phi+S \frac{d\left(\Theta^{\top} \Phi\right)}{d t} \geq-Q(e-\Delta)-A_{c}^{\top}\left(P e+S \Theta^{\top} \Phi\right)
$$

Since $\Theta^{\top} \Phi$ is the linear-in-parameter matched uncertainty of $f(x)$, then by Proposition 1 and Assumption 3

$$
\begin{aligned}
\sup _{t}\left|\frac{d\left(\Theta^{\top} \Phi\right)}{d t}\right|=\sup _{t}\left|\frac{d\left(\tilde{\Theta}^{\top} \Phi\right)}{d t}+\dot{\varepsilon}(x)+\frac{d f(x)}{d t}\right| \leq \sup _{t}\left|\dot{\tilde{\Theta}}^{\top} \Phi+\tilde{\Theta}^{\top} \dot{\Phi}\right|+\sigma_{\varepsilon}+\sigma_{f} \\
\leq \sup _{t}\left|-B^{\top} p \Phi^{\top} \Gamma \Phi\right|+\sup _{t}\left|\tilde{\Theta}^{\top} \dot{\Phi}\right|+\sigma_{\varepsilon}+\sigma_{f}
\end{aligned}
$$

The first term in the last inequality of Eq. (27) is bounded since $p$ must be a stable solution to the optimal control problem and $\Phi$ is also bounded by definition. The second term is also bounded since $\tilde{\Theta}$ must be bounded if the adaptive law is stable (an assertion that will be proved later) and $\dot{\Phi}$ is bounded by the virtue of Assumption 3 . Therefore, there exists a constant vector $\sigma_{t}>0 \in \mathbb{R}^{n}$ such that

$$
\sup _{t}\left|\frac{d\left(\Theta^{\top} \Phi\right)}{d t}\right|<\sigma_{t}
$$

Equation (26) yields three equations

$$
\begin{gathered}
\dot{P}+P A_{c}+A_{c}^{\top} P+Q=0 \\
\dot{S}+P B+A_{c}^{\top} S=0
\end{gathered}
$$

subject to $P\left(t_{f}\right)=0$ and $S\left(t_{f}\right)=0$, and

$$
\Delta \leq\left|Q^{-1}\left[P B\left(\delta_{e}-\Theta^{* \top} \Phi\right)+S \sigma_{t}\right]\right|
$$

Consider an infinite time-horizon optimal control problem by letting $t_{f} \rightarrow \infty$, then $P(t) \rightarrow P(0)$ and $S(t) \rightarrow S(0)$ and the solutions of $P$ and $S$ are determined by their steady state values. Thus

$$
\begin{gathered}
P A_{c}+A_{c}^{\top} P=-Q \\
S=-A_{c}^{-\top} P B
\end{gathered}
$$

The adjoint $p$ now becomes

$$
p=P e-A_{c}^{-\top} P B \Theta^{\top} \Phi
$$

Since $\Theta^{*}$ is constant, then $\dot{\Theta}=\dot{\tilde{\Theta}}$. Upon substituting the expression of the adjoint $p$ into Eq. (24), the following adaptive law is then obtained

$$
\dot{\Theta}=-\Gamma \Phi\left(e^{\top} P-v \Phi^{\top} \Theta B^{\top} P A_{c}^{-1}\right) B
$$

where $v>0 \in \mathbb{R}$ is introduced as a weighting constant to allow for adjustments of the second term in the adaptive law. 
Remark 1: The cost function (20) could also be penalized with $\frac{1}{2} R\left\|\Theta^{\top} \Phi\right\|^{2}>0$. This would result in an additional term in the adaptive law which would then become

$$
\dot{\Theta}=-\Gamma \Phi\left(e^{\top} P B-v \Phi^{\top} \Theta B^{\top} P A_{c}^{-1} B+\Phi^{\top} \Theta R\right)
$$

$R$ then becomes an additional tuning parameter that can be used to adjust the adaptive law. Alternatively, the adaptive law could just only include the $R$ term as

$$
\dot{\Theta}=-\Gamma \Phi\left(e^{\top} P B+\Phi^{\top} \Theta R\right)
$$

The adaptive law (18) can be shown to be stable by the following theorem:

Theorem 1: The adaptive law (18) results in stable and uniformly bounded tracking error in a compact set

$$
\mathscr{S}=\left\{e \in \mathbb{R}^{n}:\|e\|>r=\frac{2 \lambda_{\max }(P)\|B\|\left(\left\|\Theta^{* \top} \Phi\right\|_{\infty}+\left\|\delta_{\varepsilon}\right\|\right)}{\lambda_{\min }(Q)}\right\}
$$

Proof:-Choose a Lyapunov candidate function

$$
V=e^{\top} P e+\operatorname{trace}\left(\Theta^{\top} \Gamma^{-1} \Theta\right)
$$

where $P$ solves Eq. (19).

Evaluating the Lie derivative of $V$ yields

$$
\dot{V} \leq e^{\top}\left(A_{c} P+P A_{c}\right) e+2 e^{\top} P B\left(\Theta^{\top} \Phi-\Theta^{* \top} \Phi+\delta_{\varepsilon}\right)-2 \operatorname{trace}\left[\Theta^{\top} \Phi\left(e^{\top} P B-v \Phi^{\top} \Theta B^{\top} P A_{c}^{-1} B\right)\right]
$$

Using the trace identity trace $\left(A^{\top} B\right)=B A^{\top}, \dot{V}$ can be written as

$$
\dot{V} \leq-e^{\top} Q e+2 e^{\top} P B\left(\Theta^{\top} \Phi-\Theta^{* \top} \Phi+\delta_{\varepsilon}\right)-2 e^{\top} P B \Theta^{\top} \Phi+2 v \Phi^{\top} \Theta B^{\top} P A_{c}^{-1} B \Theta^{\top} \Phi
$$

The sign-definiteness of the term $P A_{c}^{-1}$ is now evaluated. We recall that a general real matrix $M$ is positive (negative) definite if and only if its symmetric part $M_{S}=\frac{1}{2}\left(M+M^{\top}\right)$ is also positive (negative) definite. Then, by pre- and post-multiplication of Eq. (19) by $A_{c}^{-\top}$ and $A_{c}^{-1}$, respectively, one gets

$$
A_{c}^{-\top} P+P A_{c}^{-1}=-A_{c}^{-\top} Q A_{c}^{-1}
$$

Since $A_{c}^{-\top} Q A_{c}^{-1}>0$, we conclude that $P A_{c}^{-1}<0$. Furthermore, $P A_{c}^{-1}$ can be decomposed into a symmetric part $M=\frac{1}{2}\left(P A_{c}^{-1}+A_{c}^{-\top} P\right)=-\frac{1}{2} A_{c}^{-\top} Q A_{c}^{-1}<0$ and an anti-symmetric part $N=\frac{1}{2}\left(P A_{c}^{-1}-A_{c}^{-\top} P\right)$. Then, $\dot{V}$ becomes

$$
\dot{V} \leq-e^{\top} Q e+2 e^{\top} P B\left(-\Theta^{* \top} \Phi+\delta_{\varepsilon}\right)-v \Phi^{\top} \Theta B^{\top} A_{c}^{-\top} Q A_{c}^{-1} B \Theta^{\top} \Phi+2 v \Phi^{\top} \Theta B^{\top} N B \Theta^{\top} \Phi
$$

Letting $y=B \Theta^{\top} \Phi$ and using the property $y^{\top} N y=0$ for an anti-symmetric matrix $N, \dot{V}$ is reduced to

$$
\dot{V} \leq-e^{\top} Q e+2 e^{\top} P B\left(-\Theta^{* \top} \Phi+\delta_{\varepsilon}\right)-v \Phi^{\top} \Theta B^{\top} A_{c}^{-\top} Q A_{c}^{-1} B \Theta^{\top} \Phi
$$

and is bounded by

$$
\dot{V} \leq-\lambda_{\min }(Q)\|e\|^{2}+2 \lambda_{\max }(P)\|B\|\left(\left\|\Theta^{* \top} \Phi\right\|+\left\|\delta_{\varepsilon}\right\|\right)\|e\|-v \lambda_{\min }(Q)\left\|A_{c}^{-1} B \Theta^{\top} \Phi\right\|^{2}
$$

where $\left\|\Theta^{* \top} \Phi\right\|=\left\|\sup _{t}\left|\Theta^{* \top} \Phi\right|\right\|$.

To ensure that $\dot{V} \leq 0$, we require that

$$
-\lambda_{\min }(Q)\|e\|^{2}+2 \lambda_{\max }(P)\|B\|\left(\left\|\Theta^{* \top} \Phi\right\|+\left\|\delta_{\varepsilon}\right\|\right)\|e\| \leq 0
$$

which implies

$$
\|e\| \geq \frac{2 \lambda_{\max }(P)\|B\|\left(\left\|\Theta^{* \top} \Phi\right\|+\left\|\delta_{\varepsilon}\right\|\right)}{\lambda_{\min }(Q)}
$$


It is noted that $\dot{e} \in \mathscr{L}_{\infty}$, but $e \in \mathscr{L}_{2} \cap \mathscr{L}_{\infty}$ since

$$
\begin{aligned}
\lambda_{\min }(Q) \int_{0}^{\infty}\|e\|^{2} d t \leq V(0)-V(t \rightarrow \infty)+2 \lambda_{\max }(P)\|B\|\left(\left\|\Theta^{* \top} \Phi\right\|\right. & \left.+\left\|\delta_{\varepsilon}\right\|\right) \int_{0}^{\infty}\|e\| d t \\
& -v \lambda_{\min }(Q) \int_{0}^{\infty}\left\|A_{c}^{-1} B \Theta^{\top} \Phi\right\|^{2} d t<\infty
\end{aligned}
$$

It follows that

$$
V(t \rightarrow \infty) \leq V(0)-v \lambda_{\min }(Q) \int_{0}^{\infty}\left\|A_{c}^{-1} B \Theta^{\top} \Phi\right\|^{2} d t<\infty
$$

$V(t)$ thus decreases inside a compact set $\mathscr{S} \subset \mathbb{R}^{n}$ where

$$
\mathscr{S}=\left\{e \in \mathbb{R}^{n}:\|e\|>r=\frac{2 \lambda_{\max }(P)\|B\|\left(\left\|\Theta^{* \top} \Phi\right\|+\left\|\delta_{\varepsilon}\right\|\right)}{\lambda_{\min }(Q)}\right\}
$$

but $V(t)$ increases inside the complementary set $\mathscr{C}=\left\{e \in \mathbb{R}^{n}:\|e\| \leq r\right\}$, which contains $e=0$, whose trajectories will all stay inside of $\mathscr{C}$. It follows by LaSalle's extensions of the Lyapunov method that $e$ is uniformly bounded and so is $\Theta$.

Remark 2: The effect of the added term in the present adaptive law is to add damping to the weight update law so as to reduce high-frequency oscillations in the weights. The damping term requires persistent excitation (PE) which is defined by the product term $\Phi \Phi^{\top}$. With persistent excitation, the weight $\Theta$ is exponentially stable and bounded. This scheme is contrasted to the well-known $\sigma_{-}{ }^{17}$ and $\varepsilon_{-}^{-16}$ modification methods and other variances which also add damping terms to prevent parameter drift in the absence of persistent excitation. ${ }^{16}$ These adaptive laws are compared as follows:

\begin{tabular}{|c|c|}
\hline Modification & Adaptive Law \\
\hline \hline$\sigma-$ & $\dot{\Theta}=-\Gamma\left(\Phi e^{\top} P B+\sigma \Theta\right), \sigma>0$ \\
\hline$\varepsilon-$ & $\dot{\Theta}=-\Gamma\left(\Phi e^{\top} P B+\mu\left\|e^{\top} P B\right\| \Theta\right), \mu>0$ \\
\hline Optimal & $\dot{\Theta}=-\Gamma\left(\Phi e^{\top} P B-v \Phi \Phi^{\top} \Theta B^{\top} P A_{c}^{-1} B\right), v>0$ \\
\hline
\end{tabular}

Table 1 - Modifications to MRAC Law

In the presence of fast adaptation, i.e., $\lambda_{\min }(\Gamma) \gg 1$, the adaptive law (18) is robustly stable with all closed-loop poles having negative real values if $v=1$. This fact can be established in the following theorem:

Theorem 2: For large adaptive gain $\Gamma$ and $\Phi^{\top} \Phi>0$ which implies PE, the adaptive law (18) results in robustly stable closed-loop tracking error dynamics

$$
\dot{e} \leq-P^{-1} Q e+B\left(\sup _{t}\left|\Theta^{* \top} \Phi\right|+\delta_{\varepsilon}\right)
$$

when $v=1$.

Proof: The adaptive law (18) can be written as

$$
\Gamma^{-1} \dot{\Theta}=-\Phi\left(e^{\top} P-v \Phi^{\top} \Theta B^{\top} P A_{c}^{-1}\right) B
$$

If $\Gamma \gg 1$ is large, then in the limit as $\Gamma \rightarrow \infty$

$$
e^{\top} P-v \Phi^{\top} \Theta B^{\top} P A_{c}^{-1}=0
$$

Solving for $B \Theta^{\top} \Phi$ yields

$$
B \Theta^{\top} \Phi=\frac{1}{v} P^{-1} A_{c}^{\top} P e
$$

$$
7 \text { of } 18
$$


Hence, the closed-loop tracking dynamics become

$$
\dot{e}=\left(A_{c}+\frac{1}{v} P^{-1} A_{c}^{\top} P\right) e+B\left(\varepsilon-\Theta^{* \top} \Phi\right) \leq\left(A_{c}+\frac{1}{v} P^{-1} A_{c}^{\top} P\right) e+B\left(\sup _{t}\left|\Theta^{* \top} \Phi\right|+\delta_{\varepsilon}\right)
$$

which, upon some algebra, can also be written as

$$
\dot{e} \leq-P^{-1}\left[Q-\left(\frac{1}{v}-1\right) A_{c}^{\top} P\right] e+B\left(\sup _{t}\left|\Theta^{* \top} \Phi\right|+\delta_{\varepsilon}\right)
$$

$A_{c}^{\top} P$ can be decomposed into a symmetric part $\frac{1}{2}\left(A_{c}^{\top} P+P A_{c}\right)=-\frac{1}{2} Q$ and an anti-symmetric part $\frac{1}{2} S=\frac{1}{2}\left(A_{c}^{\top} P-P A_{c}\right)$. The tracking error dynamics now become

$$
\dot{e} \leq-P^{-1}\left[\left(\frac{1+v}{2 v}\right) Q-\left(\frac{1-v}{2 v}\right) S\right] e+B\left(\sup _{t}\left|\Theta^{* \top} \Phi\right|+\delta_{\varepsilon}\right)
$$

The eigenvalues of $Q$ are all real positive values and those of $S$ are purely imaginary. The system is stable for all values of $v$. If $v \leq 1$, the closed-loop complex-conjugate poles move further into the left-half plane and $\operatorname{Im}[s]$ increases with decreasing $v$. In the limit, when $v \rightarrow 0$ and the adaptive law is reverted to the standard MRAC law, then $\operatorname{Im}[s] \rightarrow \infty$ which illustrates a well-known fact that fast adaptation with the standard MRAC law results in high frequency signals which can potentially lead to instability in the presence of time delay or unmodeled dynamics. Conversely, if $v$ becomes large, the effect of adaptation is reduced and in the limit when $v \rightarrow \infty$, adaptation ceases as the adaptive law (18) becomes infinitely stiff.

A special case of $v=1$ is considered. The closed-loop poles are all real, negative values with $\operatorname{Re}[s]=-\lambda\left(P^{-1} Q\right)$. The system transfer function matrix $H(s)=\left(s I+P^{-1} Q\right)^{-1}$ is strictly positive real (SPR) since $H(j \omega)+H^{\top}(-j \omega)>$ 0 , and thus the system is minimum phase and dissipative. ${ }^{18}$ The Nyquist plot of a strictly stable transfer function is strictly in the right half plane with a phase shift less than or equal to $\frac{\pi}{2} .18$

Remark 3: The adaptive law for fast adaptation results in a LTI representation of the tracking error dynamics in the limit when $\Gamma \rightarrow \infty$. This is a useful feature of this adaptive law that can enable the stability of the system to be analyzed using traditional linear control methods.

The adaptive law (18) causes the tracking error to tend to zero as $v \rightarrow 0$ if $\gamma \rightarrow \infty$ for fast adaptation. On the other hand, stability robustness requires $v>0$. Thus, a trade-off between tracking performance and stability robustness exists and, consequently, $v$ becomes a design parameter to be chosen to satisfy control requirements if $\Gamma$ is large and the input is PE. This can be shown as follows:

Lemma 1: The equilibrium state $y=0$ of the differential equation

$$
\dot{y}=-\Phi^{\top}(t) \Gamma \Phi(t) y
$$

where $y(t):[0, \infty) \rightarrow \mathbb{R}, \Phi(t) \in \mathscr{L}_{2}:[0, \infty) \rightarrow \mathbb{R}^{n}$ is a piecewise-continuous and bounded function, and $\Gamma>0 \in \mathbb{R}^{n \times n}$, is uniformly asymptotically stable, if there exists a constant $\gamma>0$ such that

$$
\frac{1}{T_{0}} \int_{t}^{t+T_{0}} \Phi^{\top}(\tau) \Gamma \Phi(\tau) d \tau \geq \gamma
$$

which implies that $y$ is bounded by a linear differential equation

$$
\dot{y} \leq-\gamma y
$$

Proof: Choose a Lyapunov candidate function

$$
\begin{gathered}
V=\frac{1}{2} y^{2} \\
\dot{V}=-\Phi^{\top}(t) \Gamma \Phi(t) y^{2}=-2 \Phi^{\top}(t) \Gamma \Phi(t) V
\end{gathered}
$$

Then, there exists $\gamma>0$ for which $V$ is uniformly asymptotically stable since

$$
V\left(t+T_{o}\right)=V(t) \exp \left(-2 \int_{t}^{t+T_{0}} \Phi^{\top}(\tau) \Gamma \Phi(\tau) d \tau\right) \leq V(t) e^{-2 \gamma T_{0}}
$$


This implies that

$$
\exp \left(-2 \int_{t}^{t+T_{0}} \Phi^{\top}(\tau) \Gamma \Phi(\tau) d \tau\right) \leq e^{-2 \gamma T_{0}}
$$

Thus, the equilibrium $y=0$ is uniformly asymptotically stable if

$$
\frac{1}{T_{0}} \int_{t}^{t+T_{0}} \Phi^{\top}(\tau) \Gamma \Phi(\tau) d \tau \geq \gamma
$$

provided $\Phi(t) \in \mathscr{L}_{2}$ is bounded.

Then $y(t) \in \mathscr{L}_{2} \cap \mathscr{L}_{\infty}$ since

$$
V(t \rightarrow \infty)-V(0) \leq-2 \gamma \int_{0}^{\infty} y^{2}(t) d t \Rightarrow 2 \gamma \int_{0}^{\infty} y^{2}(t) d t \leq V(0)-V(t \rightarrow \infty)<\infty
$$

It follows that

$$
y\left(t+T_{0}\right)=y(t) \exp \left(-\int_{t}^{t+T_{0}} \Phi^{\top}(\tau) \Gamma \Phi(\tau) d \tau\right) \leq y(t) e^{-\gamma T_{0}}
$$

which is equivalent to

$$
\dot{y}=-\Phi^{\top}(t) \Gamma \Phi(t) y \leq-\gamma y
$$

Now, suppose that $\Phi=\Phi(y(t))$, Eq. (67) still applies. The condition $\Phi(y(t)) \in \mathscr{L}_{2}$ is satisfied since $y \in \mathscr{L}_{2} \cap \mathscr{L}_{\infty}$. To show this, we first evaluate $\dot{V}$ as

$$
\dot{V}=-\Phi^{\top}(y(t)) \Gamma \Phi(y(t)) y^{2}
$$

which upon integration yields

$$
V\left(t+T_{o}\right)=V(t) \exp \left(-2 \int_{t}^{t+T_{0}} \Phi^{\top}(y(\tau)) \Gamma \Phi(y(\tau)) d \tau\right) \leq V(t) e^{-2 \gamma T_{0}}
$$

Thus, $V$ is uniformly asymptotically stable. This condition is also equivalent to

$$
\dot{V} \leq-2 \gamma V \Rightarrow y \dot{y} \leq-\gamma y^{2}
$$

which then implies Eq. (67).

Example: Consider $\phi(t)=(t+1)^{-1} \in \mathscr{L}_{2} \cdot \gamma$ is evaluated as

$$
\gamma=\frac{1}{T_{0}} \int_{0}^{T_{0}} \phi^{2}(\tau) d \tau=\frac{1}{T_{0}+1}
$$

The solutions of $\dot{y}=-y \phi^{2}(t)$ and $\dot{y}_{1}=-\gamma y_{1}$ with $y(0)=y_{1}(0)$ are

$$
\begin{gathered}
y(t)=y(0) \exp \left(-\frac{t}{t+1}\right) \\
y_{1}(t)=y(0) \exp \left(-\frac{t}{T_{0}+1}\right)
\end{gathered}
$$

Clearly, $y \leq y_{1}$ since $T_{0} \geq t$. Therefore, $\dot{y} \leq \dot{y}_{1}$.

Lemma 1 is a version of the Comparison Lemma that allows bounds on the solution of $y(t)$ to be computed from a differential inequality without the need to compute the solution itself. ${ }^{19}$ A different version of the proof is also provided by Nadrenda and Annaswamy. ${ }^{20}$ Figure 3 illustrates various functions as compared to their linear counterparts. 

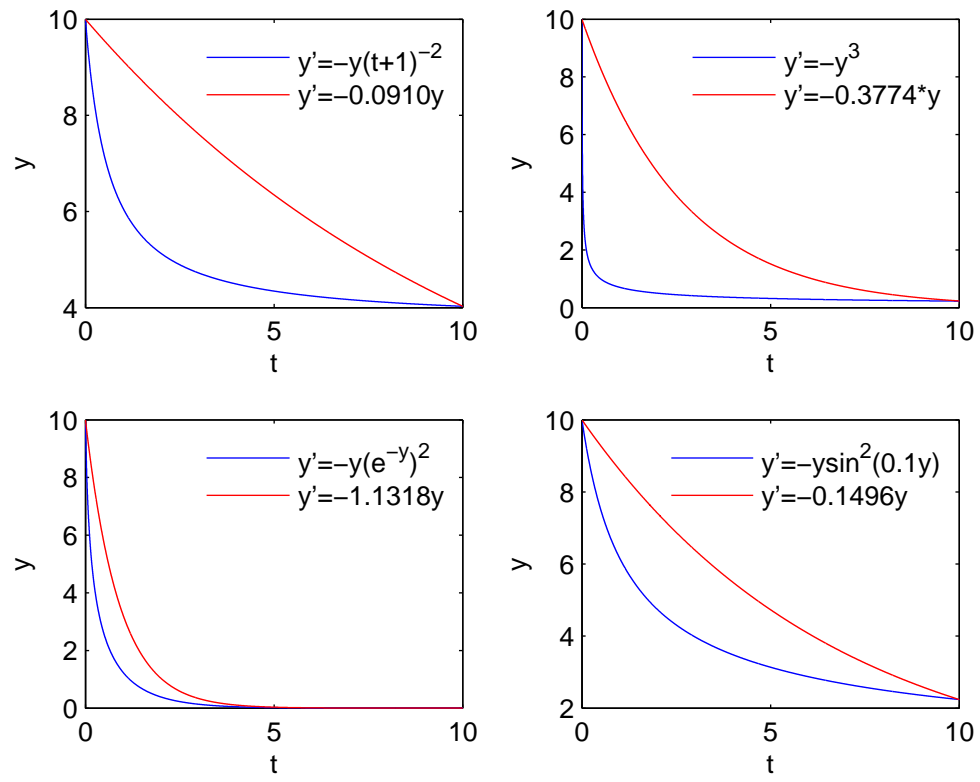

Fig. 3 - Comparison of Solutions of Differential Equations

Theorem 3: The steady state tracking error is bounded by

$$
\lim _{t \rightarrow \infty} \sup _{t}\|e\| \leq \frac{v \lambda_{\max }(P)\|B\|\left(\left\|\Theta^{* \top} \Phi\right\|_{\infty}+\left\|\delta_{\varepsilon}\right\|\right)}{\sigma_{\min }\left(A_{c}^{\top} P+v P A_{c}\right)}+\frac{\left\|A_{c}\right\|\|B\|\left(\|\beta\|+\left\|\sigma_{e}\right\|\right)}{\gamma \sigma_{\min }\left(B B^{\top}\right) \sigma_{\min }\left(A_{c}^{\top} P+v P A_{c}\right)}
$$

if there exist a constant $\gamma>0$ such that $\gamma=\inf _{t}\left(\frac{1}{T_{0}} \int_{t}^{t+T_{0}} \Phi^{\top} \Gamma \Phi\right)>0 \in \mathbb{R}$ and a constant vector $\beta>0 \in \mathbb{R}^{n}$ where $\beta=\sup _{t}\left|\tilde{\Theta}^{\top} \dot{\Phi}\right|$.

Proof: Since $e \in \mathscr{L}_{2}, x \in \mathscr{L}_{2}$, and so $\Phi(x) \in \mathscr{L}_{2}$. Using Lemma 1, the adaptive law (18) can be written as

$$
\frac{d}{d t}\left(\tilde{\Theta}^{\top} \Phi\right)=\dot{\tilde{\Theta}}^{\top} \Phi+\tilde{\Theta}^{\top} \dot{\Phi} \leq-\gamma\left(B^{\top} P e-v B^{\top} A_{c}^{-\top} P B \Theta^{\top} \Phi\right)+\beta
$$

$\beta=\sup _{t}\left|\tilde{\Theta}^{\top} \dot{\Phi}\right| \in \mathscr{L}_{\infty}$ is bounded since $\dot{\Phi} \in \mathscr{L}_{\infty}$ is bounded by Assumption 3 because

$$
\Theta^{* \top} \sup _{t}|\dot{\Phi}|=\sup _{t}\left|\dot{\varepsilon}(x)+\frac{d f(x)}{d t}\right| \leq \sup _{t}|\dot{\varepsilon}(x)|+\sup _{t}\left|\frac{d f(x)}{d t}\right| \leq \sigma_{\varepsilon}+\sigma_{f}
$$

Thus, the system dynamics with adaptation are described by

$$
\frac{d}{d t}\left[\begin{array}{c}
e \\
\tilde{\Theta}^{\top} \Phi
\end{array}\right] \leq\left[\begin{array}{cc}
A_{c} & B \\
-\gamma B^{\top} P & \gamma v B^{\top} A_{c}^{-\top} P B
\end{array}\right]\left[\begin{array}{c}
e \\
\tilde{\Theta}^{\top} \Phi
\end{array}\right]+\left[\begin{array}{c}
B \delta_{e} \\
\gamma v B^{\top} A_{c}^{-\top} P B \Theta^{* \top} \Phi+\beta
\end{array}\right]
$$

Differentiating the tracking error dynamics and upon substitution yields

$$
\ddot{e}-A_{c} \dot{e}+\gamma B B^{\top} P e-\gamma v B B^{\top} A_{c}^{-\top} P B \tilde{\Theta}^{\top} \Phi \leq B\left(\gamma v B^{\top} A_{c}^{-\top} P B \Theta^{* \top} \Phi+\beta+\sigma_{e}\right)
$$

The steady state tracking error is thus bounded by

$$
\left(\gamma B B^{\top} P+\gamma v B B^{\top} A_{c}^{-\top} P A_{c}\right) e \leq \gamma v B B^{\top} A_{c}^{-\top} P B\left(\Theta^{* \top} \Phi-\delta_{\varepsilon}\right)+B\left(\beta+\sigma_{e}\right)
$$

from which the upper bound on the norm of the steady-state tracking error is computed as

$$
\lim _{t \rightarrow \infty} \sup _{t}\|e\| \leq \frac{v \lambda_{\max }(P)\|B\|\left(\left\|\Theta^{* \top} \Phi\right\|_{\infty}+\left\|\delta_{\varepsilon}\right\|\right)}{\sigma_{\min }\left(A_{c}^{\top} P+v P A_{c}\right)}+\frac{\left\|A_{c}\right\|\|B\|\left(\|\beta\|+\left\|\sigma_{e}\right\|\right)}{\gamma \sigma_{\min }\left(B B^{\top}\right) \sigma_{\min }\left(A_{c}^{\top} P+v P A_{c}\right)}
$$


where $\sigma_{\min }$ denotes the minimum singular value.

Similarly, the steady state value and the upper bound of its norm are obtained as

$$
\begin{gathered}
-\left(\gamma v B^{\top} A_{c}^{-\top} P+\gamma B^{\top} P A_{c}^{-1}\right) B \tilde{\Theta}^{\top} \Phi \leq\left(\gamma v B^{\top} A_{c}^{-\top} P B \Theta^{* \top} \Phi+\beta+\sigma_{e}\right)+\gamma B^{\top} P A_{c}^{-1} B \delta_{e} \\
\lim _{t \rightarrow \infty} \sup _{t}\left\|\tilde{\Theta}^{\top} \Phi\right\| \leq \frac{v \lambda_{\max }(P)\left\|A_{c}\right\|\left\|\Theta^{* \top} \Phi\right\|_{\infty}}{\sigma_{\min }\left(A_{c}^{\top} P+v P A_{c}\right)}+\frac{\lambda_{\max }(P)\left\|A_{c}\right\|^{2}\left\|\delta_{\varepsilon}\right\|}{\sigma_{\min }\left(A_{c}\right) \sigma_{\min }\left(A_{c}^{\top} P+v P A_{c}\right)}+\frac{\left\|A_{c}\right\|^{2}\left(\|\beta\|+\left\|\sigma_{e}\right\|\right)}{\gamma \sigma_{\min }\left(B B^{\top}\right) \sigma_{\min }\left(A_{c}^{\top} P+v P A_{c}\right)}
\end{gathered}
$$

Thus for fast adaptation with PE, i.e., $\gamma \rightarrow \infty$, the second term on the RHS of Eq. (80) goes to zero, and the tracking error only becomes bounded and is dependent on $v$. If, in addition, $v \rightarrow 0$, then $e \rightarrow 0$, but if $v \rightarrow \infty, e \in \mathscr{L}_{\infty}$ is finite and does not tend to zero. Thus, $v$ has to be selected small enough to provide a desired tracking performance, but large enough to provide sufficient stability margins against time delay or unmodeled dynamics.

\section{Application to Flight Control}

Consider the following adaptive flight control architecture as shown in Fig. 4. The control architecture comprises: 1) a reference model that translates rate commands into desired acceleration commands, 2) a proportional-integral (PI) feedback control for rate stabilization and tracking, 3) a dynamic inversion controller that computes actuator commands using desired acceleration commands, and 4) a neural net direct MRAC due to Rysdyk and Calise ${ }^{8}$

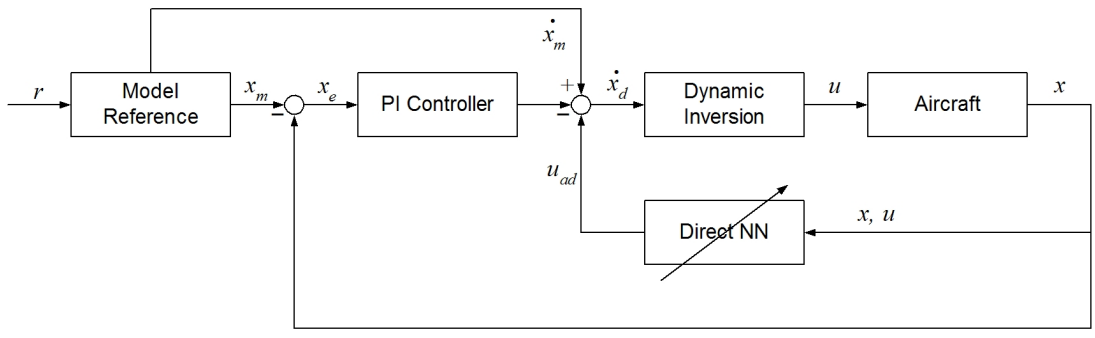

Fig. 4 - Direct Neural Network Adaptive Flight Control

Adaptive flight control can be used to provide consistent handling qualities and restore stability of aircraft under off-nominal operating conditions such as those due to failures or damage. A reduced-order equation of the linearized angular motion of a damaged aircraft can be described by

$$
\dot{x}=A x+B u+G z+f(x, u, z)
$$

where $x=\left[\begin{array}{ccc}p & q & r\end{array}\right]^{\top}$ is the angular rate vector; $u=\left[\begin{array}{ccc}\delta_{a} & \delta_{e} & \delta_{r}\end{array}\right]^{\top}$ is the control surface deflection vector; $z=\left[\begin{array}{ccc}\alpha & \beta & \delta_{T}\end{array}\right]^{\top}$ is a trim state vector; $A \in \mathbb{R}^{3 \times 3}, B \in \mathbb{R}^{3 \times 3}$, and $G \in \mathbb{R}^{3 \times 3}$ are known; and $f(x, u, z)$ represents a structured uncertainty which has the form

$$
f(x)=\Delta A x+\Delta B u+\Delta G z
$$

where $\Delta A, \Delta B$, and $\Delta G$ are changes to the $A, B$, and $G$ matrices of the aircraft linear plant model.

The objective is to design a dynamic inversion flight control law with a direct adaptive control augmentation to provide consistent handling qualities which may be specified by a reference model according to

$$
\dot{x}_{m}=A_{m} x+B_{m} r
$$

where $A_{m} \in \mathbb{R}^{3 \times 3}$ is Hurwitz, $B_{m} \in \mathbb{R}^{3 \times 3}$ is known, $r \in \mathscr{L}_{2}$ is a bounded pilot command with its time derivative $\dot{r} \in \mathscr{L}_{\infty}$ also bounded 
Let $\dot{x}_{d}$ be a desired acceleration that comprises the reference model acceleration, a proportional-integral feedback control, and a neural net adaptive signal

$$
\dot{x}_{d}=A_{m} x_{m}+B_{m} r+K_{p}\left(x_{m}-x\right)+K_{i} \int_{0}^{t}\left(x_{m}-x\right) d \tau-u_{a d}
$$

where $u_{a d}=\Theta^{\top} \Phi$ with $\Phi=\left[\begin{array}{lll}x^{\top} & u^{\top} & z^{\top}\end{array}\right]^{\top}$.

Assuming that $B$ is invertible, then the dynamic inversion controller is computed as

$$
u=B^{-1}\left(\dot{x}_{d}-A x-B u-G z\right)
$$

Computing the acceleration error yields

$$
\dot{\tilde{x}}=-K_{p} \tilde{x}-K_{i} \int_{0}^{t} \tilde{x} d \tau+u_{a d}-f(x, u)
$$

where $\tilde{x}=x_{m}-x, K_{p}=\operatorname{diag}\left(k_{p, 1}, k_{p, 2}, k_{p, 3}\right)>0$, and $K_{i}=\operatorname{diag}\left(k_{i, 1}, k_{i, 2}, k_{i, 3}\right)>0$ are matrices of the proportional and integral gains for roll, pitch, and yaw.

Defining the tracking error as

$$
e=\left[\begin{array}{c}
\int_{0}^{t} \tilde{x} d \tau \\
\tilde{x}
\end{array}\right]
$$

then the tracking error dynamics are expressed by

$$
\dot{e}=A_{c} e+b\left(u_{a d}-f\right)
$$

where

$$
\begin{gathered}
A_{c}=\left[\begin{array}{cc}
0 & I \\
-K_{i} & -K_{p}
\end{array}\right] \\
b=\left[\begin{array}{l}
0 \\
I
\end{array}\right]
\end{gathered}
$$

Let $Q=2 I$, then the solution of Eq. (19) yields

$$
P=\left[\begin{array}{cc}
K_{i}^{-1} K_{p}+K_{p}^{-1}\left(K_{i}+I\right) & K_{i}^{-1} \\
K_{i}^{-1} & K_{p}^{-1}\left(I+K_{i}^{-1}\right)
\end{array}\right]>0
$$

$A_{c}^{-1}$ is computed to be

$$
A_{c}^{-1}=\left[\begin{array}{cc}
-K_{i}^{-1} K_{p} & -K_{i}^{-1} \\
I & 0
\end{array}\right]
$$

Evaluating the term $b^{\top} P A_{c}^{-1} b$ yields

$$
b^{\top} P A_{c}^{-1} b=-K_{i}^{-2}<0
$$

Applying the the adaptive law (18), the weight update law is then given by

$$
\dot{\Theta}=-\Gamma \Phi\left(e^{\top} P b+v \Phi^{\top} \Theta K_{i}^{-2}\right)
$$

Thus, the damping term in the adaptive law only depends on the integral gain $K_{i}$.

A simulation of pitch rate doublet is performed to illustrate the adaptive law (18) with the optimal control modification. The uncertainty is due to airframe structural damage which in this case represents a $25 \%$ loss of the left wing of a generic transport model (GTM) as shown in Fig. 5. 


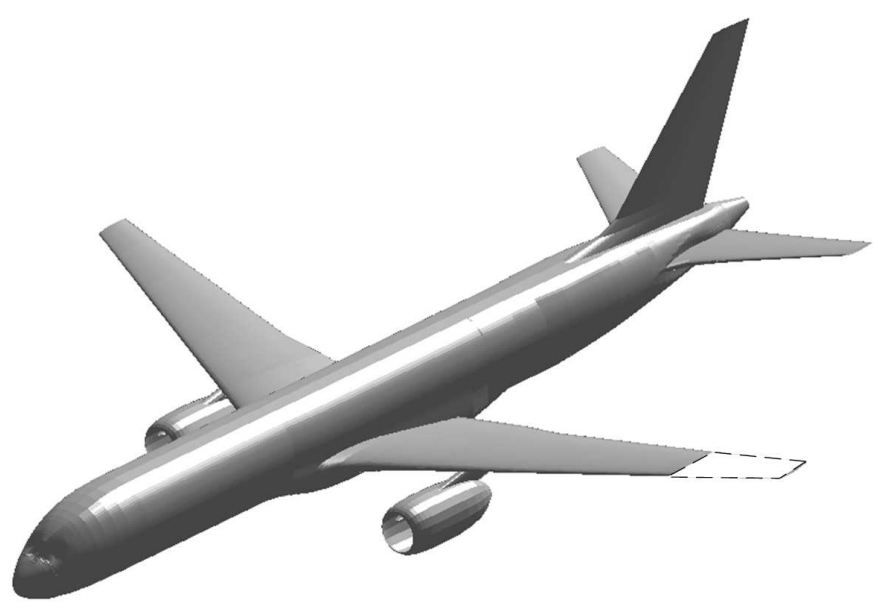

Fig. 5 - Damaged Generic Transport Model

Figure 6 is a plot of the aircraft angular rates with only PI control and without adaptive control. Due to the asymmetric damage, a pitch rate command results in both roll and yaw rate responses due to cross-coupling effects. The response is completely unacceptable due to the excessive roll and yaw rates.
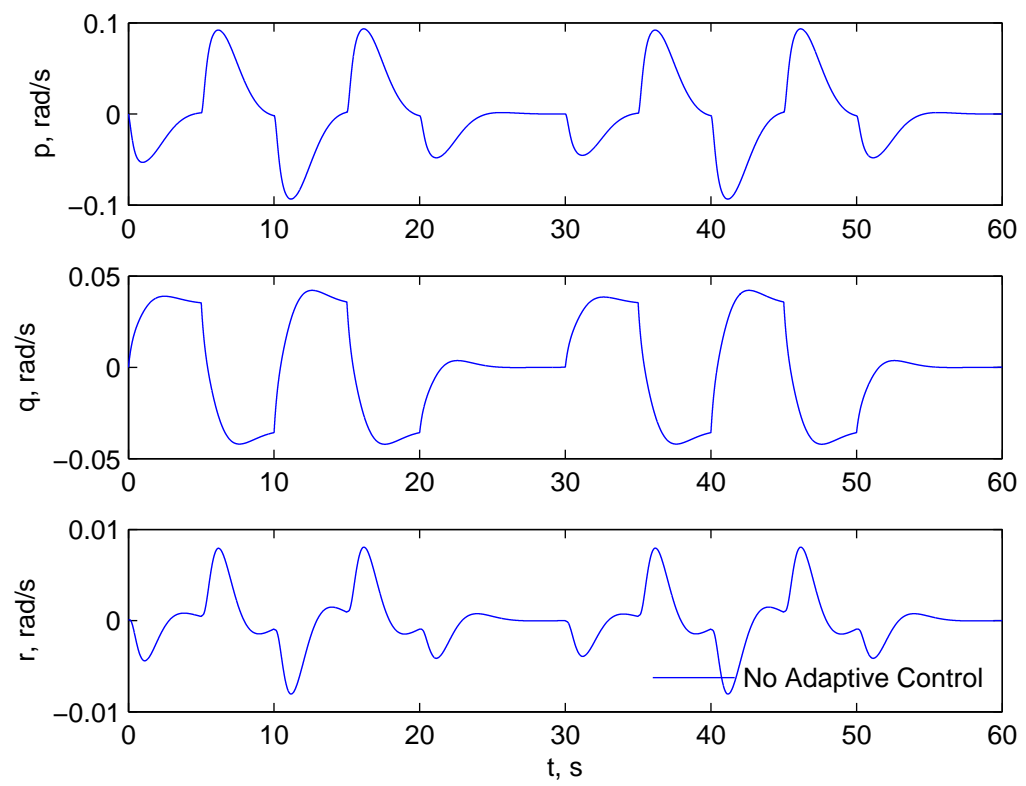

Fig. 6 - Aircraft Rate Response with PI Control

Figure 7 is a plot of the aircraft angular rates due to the standard direct MRAC $(v=0)$ using a learning rate $\Gamma=10^{4}$. The tracking performance drastically improves in all axes. However, high-frequency oscillations can clearly be seen in the yaw rate response and to a much lesser extent in the pitch and roll channels.. Further increase in the learning rate results in progressively larger high frequency amplitudes and eventually leads to a numerical instability when $\Gamma>2 \times 10^{4}$ due to a sampling limitation. 

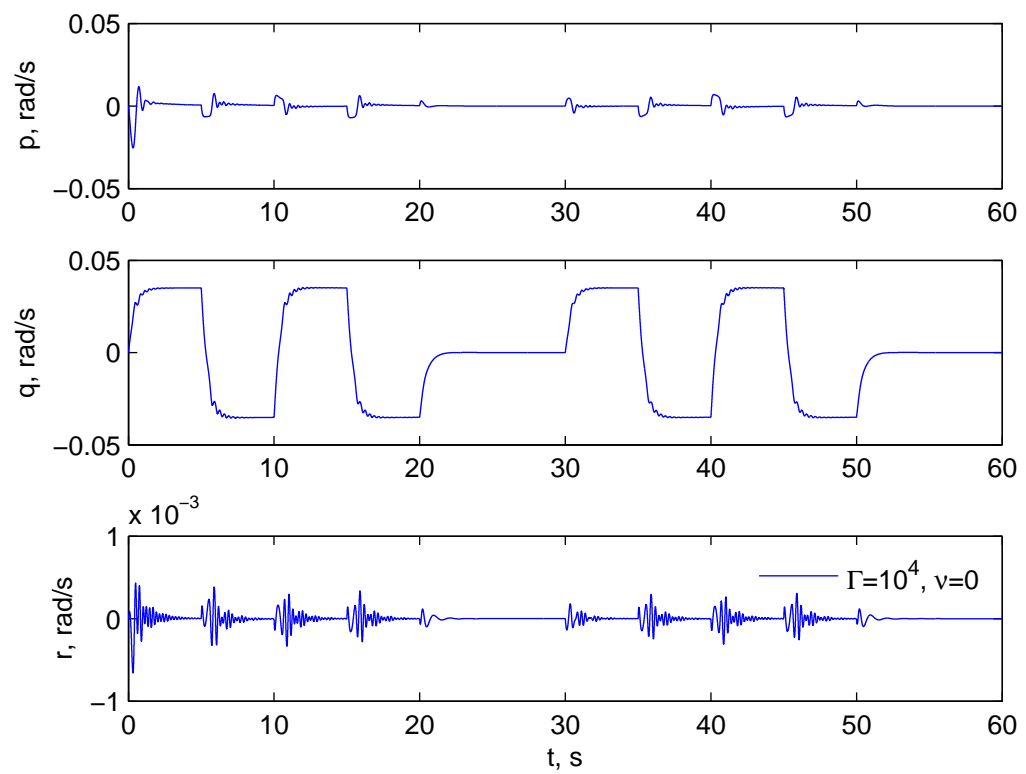

Fig. 7 - Aircraft Rate Response with Standard MRAC

In contrast, the aircraft rate response for the optimal control modification tracks the reference model very well as can be seen in Fig. 8. Furthermore, the optimal control modification results in no observable high-frequency oscillation in spite of the fact that the learning rate is two orders of magnitude greater than that for the standard direct MRAC. For this simulation, a value of $v=0.033$ is used. A larger value of $v$ will degrade the tracking performance but improve stability robustness. For comparison, the simulation also includes the $\varepsilon$-modification as shown in Fig. 9. A value of $\mu=0.25$ is used with a learning rate $\Gamma=10^{4}$. The $\varepsilon$-modification significantly reduces the high frequency in the yaw rate response, but at the expense of the tracking performance as the amplitudes in the roll and yaw channels significantly increase.
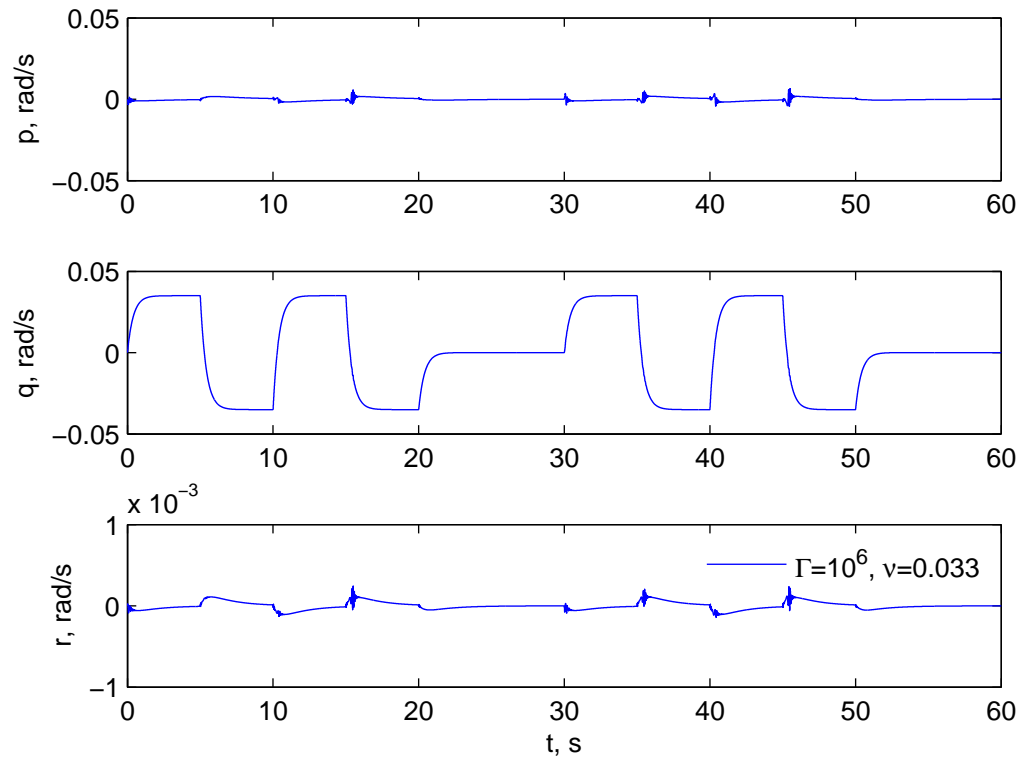

Fig. 8- Aircraft Rate Response with Optimal Control Modification 

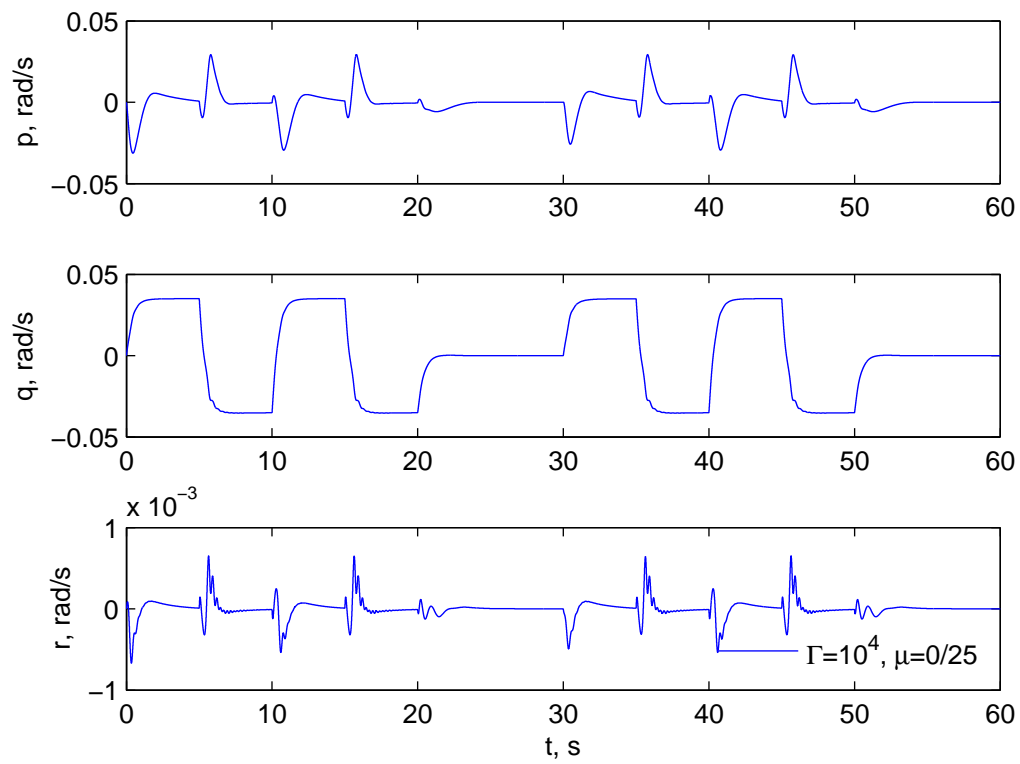

Fig. 9- Aircraft Rate Response with $\varepsilon$-Modification

The simulation illustrates a potential benefit of the optimal control modification for fast adaptation. In practice, there is a practical limit of how large a learning rate would be. In general, actuator dynamics can impose constraints on the learning rate. The frequency separation between the adaptation and actuator dynamics can lead to potential problems. Nonetheless, the optimal control modification demonstrates the tolerance to larger learning rates than the standard MRAC which can be beneficial when fast adaptation is needed to deal with large uncertainties.

One of the issues with adaptive control is the lack of metrics to assess stability robustness in the presence of unmodeled dynamics and or time delay. With fast adaptation, it is known that the direct MRAC results in reduced phase and time-delay margins. ${ }^{21}$ Thus, the learning rate must be chosen carefully in order to avoid instability due to time delay and unmodeled dynamics. The optimal control modification is shown to provide more robustness when $v$ approaches unity. Hence, it can also increase a system's tolerance to destabilizing uncertainties like time delay.

An approximate, simple method for analyzing the stability margin of the optimal control modification is presented. Toward that end, the tracking error dynamics can be expressed as

$$
\dot{x}_{m}-\dot{x}=b^{\top} \dot{e} \leq b^{\top} A e+b^{\top} b\left(\tilde{\Phi}^{\top} \Theta+\delta_{\varepsilon}\right)
$$

From Eq. (75), we get

$$
\tilde{\Theta}^{\top} \Phi \leq\left(s+\gamma v K_{i}^{-2}\right)^{-1}\left(-\gamma b^{\top} P e+\frac{\gamma \nu K_{i}^{-2}\left\|\Theta^{* \top} \Phi\right\|_{\infty}}{s}+\frac{\beta}{s}\right)
$$

where $\gamma \leq \int \Phi^{\top} \Gamma \Phi d \tau>0 \in \mathbb{R}$ according to Lemma 1 .

Substituting Eq. (98) into Eq. (97) yields

$$
\begin{aligned}
A_{m} x_{m}+B_{m} r-s x \leq-\left[\left(\frac{K_{i}}{s}+K_{p}\right)+\gamma\left(s+\gamma v K_{i}^{-2}\right)^{-1}\left(\frac{P_{12}}{s}+P_{22}\right)\right] \tilde{x} & \\
& +\left(s+\gamma v K_{i}^{-2}\right)^{-1}\left(\frac{\gamma \nu K_{i}^{-2}\left\|\Theta^{* \top} \Phi\right\|_{\infty}}{s}+\frac{\beta}{s}\right)+\delta_{\varepsilon}
\end{aligned}
$$

where $P_{12}=K_{i}^{-1}=\operatorname{diag}\left(k_{i, 1}^{-1}, k_{i, 2}^{-1}, k_{i, 3}^{-1}\right)$, and $P_{22}=K_{p}^{-1}\left(I+K_{i}^{-1}\right)=\operatorname{diag}\left(k_{p, 1}^{-1}+k_{p, 1}^{-1} k_{i, 1}^{-1}, k_{p, 2}^{-1}+k_{p, 2}^{-1} k_{i, 2}^{-1}, k_{p, 3}^{-1}+k_{p, 3}^{-1} k_{i, 3}^{-1}\right)$.

Thus, the loop transfer function matrix from $\tilde{x}$ to $x$ is then obtained as

$$
H(s)=\frac{1}{s}\left[\left(\frac{K_{i}}{s}+K_{p}\right)+\gamma\left(s+\gamma v K_{i}^{-2}\right)^{-1}\left(\frac{P_{12}}{s}+P_{22}\right)\right]
$$


which can be broken into individual loop transfer functions since $K_{p}, K_{i}, P_{12}$, and $P_{22}$ are all diagonal matrices which imply $H(s)$ is also diagonal whose elements are

$$
h_{j}(s)=\frac{k_{p, j} s^{2}+\left(k_{i, j}+\gamma v k_{p, j} k_{i, j}^{-2}+\gamma p_{12, j}\right) s+\gamma v k_{i, j}^{-1}+\gamma p_{22, j}}{s^{3}+\gamma v k_{i, j}^{-2} s^{2}}, j=1,2,3
$$

Figure 10 is a plot of the phase margin of $h_{2}(s)$ for the pitch rate as a function of $v$ for different values of $\gamma$. Increasing $v$ is shown to result in improved phase margin. Increasing $\gamma$ causes the phase margin at $v=0$ to approach to zero, but moves the phase margin closer to 90 degrees for $v>0$. The margin at $v=1$ is not necessarily the greatest. For large values of $\gamma$, this occurs at some values of $v<1$, but the differences are small from that at $v=1$. The phase margin converges asymptotically to its value corresponding to the non-adaptive PI control when $v$ is large. Theoretically, the ideal time-delay margin of this transfer function is infinite. Realistically, it is expected that the optimal control modification would provide an increase in the time-delay margin, which is proportional to the phase margin.

It should be noted that Fig. 10 should be viewed in a relative sense rather than an absolute sense. The key research question is how to select $T_{0}$ which is a time window in which the parameter $\gamma$ is to be computed. In Fig. 10, $\gamma$ is computed for the entire time interval which may provide unrealistic estimates of stability margins. One research idea has been suggested to adjust the learning rate or in this case the parameter $v$ periodically by evaluating $\gamma$ for a moving time window during which the system is approximately bounded by an equivalent LTI system based on the Comparison Lemma. ${ }^{21}$

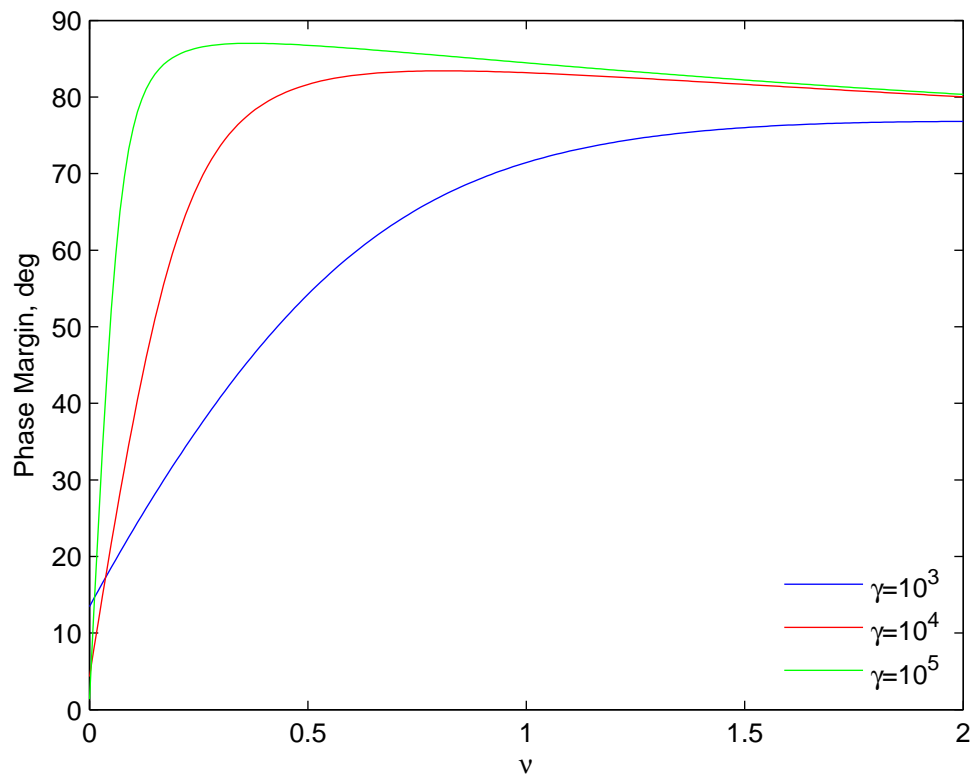

Fig. 10 - Phase Margin Analysis of Optimal Control Modification

Figure 11 illustrates the time delay effect on the optimal control modification. A time delay is introduced between the aircraft plant input and output to simulate destabilizing uncertainties. For the same learning rate $\Gamma=10^{4}$, the standard MRAC can tolerate up to $0.004 \mathrm{~s}$ time delay before the adaptive law goes unstable. With the optimal control modification, the time delay margin increases to $0.010 \mathrm{~s}$ and $0.114 \mathrm{~s}$ for $v=0.033$ and $v=0.33$, respectively. Thus, this is consistent with the observation above that increasing $v$ results in improved stability margins. However, this would come at the expense of tracking performance which would become worse as $v$ increases. 

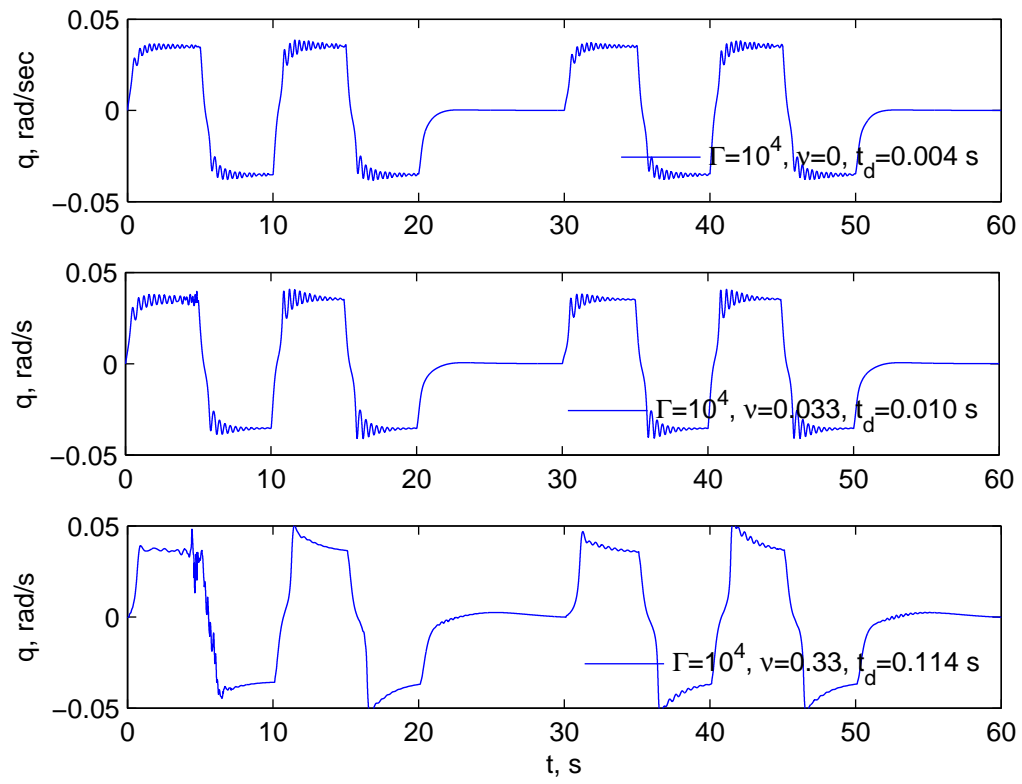

Fig. 11- Pitch Rate Responses with Time Delay

\section{Conclusions}

This study presents a new modification to the standard model-reference adaptive control based on an optimal control formulation of minimizing the norm of the tracking error. The modification adds a damping term to the adaptive law that is proportional to the persistent excitation. The modification enables fast adaptation without sacrificing robustness. When the learning rate tends to a very large value, the tracking error dynamics become approximately linear in a bounded sense. This is a useful feature that can allow stability of the adaptive law to be studied in the context of linear time invariant systems. The modification can be tuned using a parameter $v$ to provide a trade-off between tracking performance and stability robustness. Increasing $v$ results in better stability margins but reduced tracking performance. When $v$ approaches unity, the system has a phase shift close to 90 degrees. Simulations demonstrate the effectiveness of the modification, which shows that tracking performance can be achieved at a much larger learning rate than the standard model-reference adaptive control and that the adaptive law can tolerate a much greater time delay in the system.

\section{References}

${ }^{1}$ Totah, J., Krishnakumar, K., and Vikien, S., "Integrated Resilient Aircraft Control - Stability, Maneuverability, and Safe Landing in the Presence of Adverse Conditions", NASA Aeronautics Research Mission Directorate Aviation Safety Program, April 13, 2007.

${ }^{2}$ Bosworth, J. and Williams-Hayes, P.S., "Flight Test Results from the NF-15B IFCS Project with Adaptation to a Simulated Stabilator Failure”, AIAA Infotech@ Aerospace Conference, AIAA-2007-2818, 2007.

${ }^{3}$ Sharma, M., Lavretsky, E., and Wise, K., "Application and Flight Testing of an Adaptive Autopilot On Precision Guided Munitions", AIAA Guidance, Navigation, and Control Conference, AIAA-2006-6568, 2006.

${ }^{4}$ Jenkins, D.R., "Hypersonics before the Shuttle: A Concise History of the X-15 Research Airplane", NASA Special Publication, SP-20004518, June 2000, Monographs in Aerospace History: No. 18.

${ }^{5}$ Steinberg, M.L., "A Comparison of Intelligent, Adaptive, and Nonlinear Flight Control Laws", AIAA Guidance, Navigation, and Control Conference, AIAA-1999-4044, 1999.

${ }^{6}$ Rohrs, C.E., Valavani, L., Athans, M., and Stein, G., "Robustness of Continuous-Time Adaptive Control Algorithms in the Presence of Unmodeled Dynamics", IEEE Transactions on Automatic Control, Vol AC-30, No. 9, pp. 881-889, 1985.

${ }^{7}$ Eberhart, R.L. and Ward, D.G., "Indirect Adaptive Flight Control System Interactions", International Journal of Robust and Nonlinear Control, Vol. 9, pp. 1013-1031, 1999.

${ }^{8}$ Rysdyk, R.T. and Calise, A.J., "Fault Tolerant Flight Control via Adaptive Neural Network Augmentation", AIAA Guidance, Navigation, and Control Conference, AIAA-1998-4483, 1998.

${ }^{9}$ Kim, B.S. and Calise, A.J., "Nonlinear Flight Control Using Neural Networks", Journal of Guidance, Control, and Dynamics, Vol. 20, No. 1, pp. 26-33, 1997. 
${ }^{10}$ Johnson, E.N., Calise, A.J., El-Shirbiny, H.A., and Rysdyk, R.T., "Feedback Linearization with Neural Network Augmentation Applied to X-33 Attitude Control", AIAA Guidance, Navigation, and Control Conference, AIAA-2000-4157, 2000.

${ }^{11}$ Idan, M., Johnson, M.D., and Calise, A.J., "A Hierarchical Approach to Adaptive Control for Improved Flight Safety", AIAA Journal of Guidance, Control and Dynamics, Vol. 25, No. 6, pp. 1012-1020, 2002.

${ }^{12}$ Cao, C., Patel, V.V., Reddy, C.K., Hovakimyan, N., Lavretsky, E., and Wise, K., "Are Phase and Time-Delay Margin Always Adversely Affected by High Gains?", AIAA Guidance, Navigation, and Control Conference, AIAA-2006-6347, 2006.

${ }^{13}$ Idan, M., Johnson, M.D., Calise, A.J., and Kaneshige, J., "Intelligent Aerodynamic/Propulsion Flight Control For Flight Safety: A Nonlinear Adaptive Approach", American Control Conference, Arlington, VA, June 2001.

${ }^{14}$ Hovakimyan, N., Kim, N., Calise, A.J., Prasad, J.V.R., and Corban, E.J., "Adaptive Output Feedback for High-Bandwidth Control of an Unmanned Helicopter", AIAA Guidance, Navigation and Control Conference, AIAA-2001-4181, 2001.

${ }^{15}$ Nguyen, N., Krishnakumar, K., Kaneshige, J., and Nespeca, P., "Dynamics and Adaptive Control for Stability Recovery of Damaged Asymmetric Aircraft", AIAA Guidance, Navigation, and Control Conference, AIAA-2006-6049, 2006.

${ }^{16}$ Narendra, K.S. and Annaswamy, A.M., "A New Adaptive Law for Robust Adaptation Without Persistent Excitation", IEEE Transactions on Automatic Control, Vol. AC-32, No. 2, pp. 134-145, 1987.

${ }^{17}$ Ioannu, P.A. and Sun, J. Robust Adaptive Control, Prentice-Hall, 1996.

${ }^{18}$ Slotine, J.-J. E. and Li W., Applied Nonlinear Control, Prentice-Hall, 1991.

${ }^{19}$ Khalil, H.K., Nonlinear Systems, Prentice-Hall, 2002.

${ }^{20}$ Narendra, K.S. and Annaswamy, A.M., Stable Adaptive Systems, Dover Publications, 2005.

${ }^{21}$ Nguyen, N. and Boskovic, J., "Bounded Linear Stability Margin Analysis of Nonlinear Hybrid Adaptive Control”, Proceeding of 2008 IEEE American Control Conference, June 2008. 